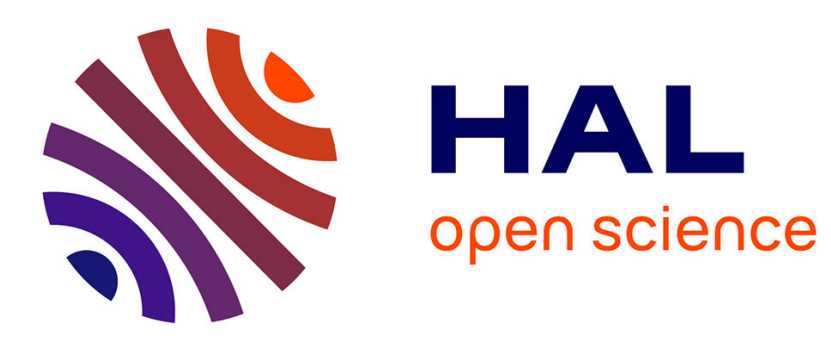

\title{
No future. Grammaire du sujet postmoderne
}

Jean-Jacques Rassial, Michèle Benhaim

\section{To cite this version:}

Jean-Jacques Rassial, Michèle Benhaim. No future. Grammaire du sujet postmoderne. L'Évolution Psychiatrique, 2009. hal-01429429

\section{HAL Id: hal-01429429 \\ https://hal-amu.archives-ouvertes.fr/hal-01429429}

Submitted on 10 Jan 2017

HAL is a multi-disciplinary open access archive for the deposit and dissemination of scientific research documents, whether they are published or not. The documents may come from teaching and research institutions in France or abroad, or from public or private research centers.
L'archive ouverte pluridisciplinaire HAL, est destinée au dépôt et à la diffusion de documents scientifiques de niveau recherche, publiés ou non, émanant des établissements d'enseignement et de recherche français ou étrangers, des laboratoires publics ou privés. 


\section{Jean-Jacques Rassial Michèle Benhaïm}

\section{No future \\ Grammaire du sujet postmoderne}

Le malaise contemporain, affectant en particulier la suite des générations, et les promesses des surmois œdipien et culturel $^{1}$ (mal traduit par surmoi « collectif»), se joue dans la temporalité autant que dans la spatialité. Les deux sempiternelles questions, « d'où je viens ? » et « où je vais ? » en témoignent ; mais en deçà de cette subjectivation, dans un espace qui a changé ${ }^{2}$, et dans une histoire centrée paradoxalement sur le présent, où la mort et la disparition, dans un démenti relatif à l'effet de la Shoah, sont renvoyées à une affaire personnelle, ce sont les catégories d'espace et de temps qui ont perdu leur caractère apriorique. C'est à partir de la langue, dimension commune au sujet et à la culture, que nous aborderons la construction de ces catégories.

Enfin, nous tâcherons de montrer qu'en réalité il y a effacement de la promesse œdipienne, promesse d'une jouissance toujours à venir, se déclinant au futur antérieur sous la forme exemplaire « quand tu auras renoncé

\footnotetext{
Jean-Jacques Rassial, professeur des Universités, directeur du Laboratoire de psychanalyse et psychopathologie clinique (LPPC) EA 3278, université d'Aix-Marseille, 3 place Victor Hugo, F- 13001 Marseille. Michèle Benhaïm, maître de conférences, habilitée à diriger des recherches, membre du LPPC, université d'Aix-Marseille ; 8 rue Alfred de Musset, F- 13006 Marseille.

1. Le concept de "surmoi culturel », mentionné seulement dans Malaise dans la civilisation, parcourt en fait toute l'œuvre anthropologique de Freud, depuis « Morale sexuelle civilisée... », p. 33, jusqu'à Moïse et le monothéisme, p. 224.

En 1930, dans Le malaise dans la culture, on retrouve le travail culturel, affaire des hommes contraints à des sublimations pulsionnelles croissantes, producteur d'un surmoi culturel exprimant ses exigences par une éthique réglant les relations sociales.

L'instauration d'un surmoi culturel, instance appartenant à la psyché de masse, selon Freud, produirait des idéaux culturels et élèverait des exigences. Parmi ces dernières, l'éthique regroupe celles qui concernent les relations sociales. Elle participerait avec le surmoi à la neutralisation de la pulsion d'agression.

2. J.-J. Rassial, «L'espace adolescent : du monde clos à l'univers infini », dans Sortir : l'opération adolescente, Toulouse, érès, 2000.
} 
à la jouissance, tu y auras droit plus tard ». La tromperie contenue dans cet énoncé aboutit à une disqualification parentale (parents à l'origine de la duperie) structurante du " passage » à l'adolescence ${ }^{3}$. Cette disparition conduit à ce que le futur antérieur, temps de la promesse, ne soutient plus le sujet. Ainsi, la promesse implicite du surmoi culturel à l'adolescence, " quand tu auras obtenu ton diplôme, tu auras du travail », est aujourd'hui un énoncé qui se conjugue non plus au mode indicatif mais au conditionnel, accentuant le caractère hypothétique du projet scolaire de l'adolescent.

\section{LE TEMPS ET LA LANGUE}

Posons les prémices de cette réflexion : La langue, propose Lacan, est la condition de l'inconscient ; l'idée immédiatement associée à cette proposition fait de l'inconscient le lieu du sujet ${ }^{4}$. Parler de la langue, que Lacan écrit « lalangue » pour des raisons que nous évoquerons plus loin, et non plus du langage, accentue l'idée que le sujet, s'il est effet d'une langue, est conditionné non par une structure générale du langage, mais par un état particulier d'une langue donnée. Cela donne un statut autre à la langue " étrangère » où pourrait se dire ce qui ne réussit pas à se dire dans la langue maternelle ${ }^{5}$. Lacan, lecteur de la grammaire de Pichon et Damourette, localise le sujet de l'énonciation dans le "ne » explétif, que ce dernier se prolonge, dans la langue française spécifiquement, d'un " pas » forclusif ou d'un " que » restrictif, ou qu'il soit isolé, par exemple dans la circonstancielle subjonctive «... avant qu'il ne soit là », marquant déjà mode et temps ${ }^{6}$.

Une fois complexifié le rapport sémantique du signifiant et du signifié, Lacan dépasse le structuralisme et nous ouvre la porte d'une réflexion sur le statut syntaxique du sujet. Le débat avec Chomsky redevient alors d'actualité : si toute langue doit réguler grammaticalement tout autant le rapport « sujet-verbe-complément » que l'expression d'une temporalité (au titre du

3. J.-J. Rassial, Le passage adolescent, Toulouse, érès, 1996.

4. Lorsque Lacan avance que « l'inconscient est structuré comme un langage » et que "le signifiant (c'est ce qui) représente un sujet pour un autre signifiant », il pose l'inconscient comme lieu du sujet, c'est-à-dire qu'il n'y a de sujet que dans le discours. Ce que Freud soutenait déjà à travers l'analyse des petits évènements qui constituent sa Psychopathologie de la vie quotidienne et, bien sûr, dans L'interprétation des rêves.

5. J.-J. Rassial, "Remarques sur le verlan des beurs », dans La psychanalyse de l'enfant, $\mathrm{n}^{\circ} 6$, 1990.

6. En grammaire, à la différence du ne... pas, le ne explétif n'a pas par lui-même de sens négatif. En psychanalyse, l'analysant est en position de recherche de « sa » vérité : dans ce contexte, la signification du mot "vérité » n'a rien à voir avec une exactitude des propos tenus ou des faits relatés, puisqu'il s'agit de la vérité du désir inconscient auquel, par définition, on n'a pas accès consciemment. En outre, le désir refoulé dans l'inconscient ignore le temps et conserve donc toujours son intensité (en témoignent les symptômes). Il ignore de surcroît la négation, le doute et la contradiction, soumis à la « rationalité » des processus primaires qui l'animent. 
circonstanciel - et dès lors il importe peu de savoir si cet « automatisme » s'inscrit en même temps dans la langue et dans le cerveau), ce qui semble l'arbitraire d'une complexification de la suite " passé-présent-futur », comme celle des modes et de leur usage spécifique dans une langue, selon une concordance d'extension variable, définit autrement culture et sujet. Chaque langue possède sa logique des modes et des temps. On peut constater la pauvreté des moyens de complexifier les temps et les modes en anglais, ce qui est accentué dans l'actualité de l'usage de la langue américaine. Cette complexité est simplifiée en français moderne, mais reste intacte dans certaines langues vivantes comme le portugais, dont la grammaire reste proche du latin. C'est cette grammaire qui ordonne autrement la promesse, aussi bien sociale que moïque. L'effacement, dans la langue française actuelle, de l'imparfait du subjonctif ne saurait se réduire à un simple effet interne à la syntaxe, mais doit être pensé par rapport à l'état d'une culture.

\section{LE FUTUR ANTÉRIEUR}

La langue française comme la langue allemande disposent d'un temps particulier, le futur antérieur, absent d'autres langues. Ce futur « antérieur » est particulièrement bien nommé, puisqu'il ne s'agit pas simplement de désigner deux futurs, l'un proche et l'autre éloigné (sans cette dimension d'un passé non encore accompli, l'adjonction du verbe « aller » comme auxiliaire aurait suffi dans le français contemporain), mais de désigner le passé non encore accompli, d'un futur, sans avoir recours au conditionnel ou au subjonctif (puisque futur et passé sont deux temps de mode indicatif). On sait que Lacan suit Heidegger pour faire du futur antérieur ${ }^{7}$ le temps du sujet, proposition qui peut sembler énigmatique, mais dont nous ferons la matière de notre proposition ici.

Le temps grammatical, celui qui s'écrit, fait question pour le parlêtre : le futur antérieur est le temps du sujet en tant qu'il anticipe, dans un futur incertain et lointain, un passé encore futur dans le présent de l'énonciation et qui conditionnerait ce qui reste à advenir.

Depuis ses anciens travaux, Lacan a proposé d'envisager la temporalité du sujet comme un temps logique plus que chronologique. Le temps logique de Lacan devient la condition de l'acte. Dans l'exemple des prisonniers, nous voyons comment il noue le désir du sujet à la condition de l'acte ${ }^{8}$. Il

7. J. Lacan, « Radiophonie », Scilicet 2/3, Paris, Le Seuil, 1970, et M. Heidegger, Etre et Temps, tr. fr. F. Vezin, Paris, Gallimard, 1986 (7éd. 1998).

8. La certitude anticipée renvoie à la question du temps logique : « L'après faisait antichambre, pour que l'avant pût prendre rang. » Ce texte est un " nouveau sophisme », nouveau au sens où le sophisme classique n'intègre pas la dimension du temps. Un directeur de prison doit libérer un détenu. Il en fait venir trois et leur propose l'épreuve suivante. Cinq disques ne se distin- 
ne s'agit pas, pas plus que dans la place donnée au langage, de réviser, ni même de dépasser Freud, mais, selon la formule même de Lacan, d'un retour à Freud.

Après, et avec, Lacan, on peut interpréter la problématique freudienne de cette temporalité, dont il nous donne une clé avec les concepts d'aprèscoup et de latence, qui traversent tous ses travaux depuis l'hypothèse traumatique, en opposition à la notion plus vague $d^{\prime}$ « originaire ». Posons pour ce faire quelques éléments freudiens ${ }^{9}$ :

Premièrement, un événement ne s'avère traumatique qu'à la condition de se réaliser deux fois : la première dans la petite enfance, la seconde à la puberté. Cette condition implique un temps logique de latence, qui ne sera « psychologisé » que plus tard, et surtout par d'autres. Freud ne modifie ici sa théorie que de substituer le fantasme œdipien à la réalité de l'événement.

Deuxièmement, l'événement réel est déplacé du psychogénétique au phylogénétique, à l'origine, mythique, de la culture. L'efficience du père passant de sa réalité pour l'enfant à sa fonction symbolique dans la fondation du lien social. L'œdipe n'est plus alors pour l'enfant une invention familiale, mais un mode de régulation proposé par la culture, à l'ambivalence primaire

guent que par leur couleur : trois sont blancs et deux noirs. Certains de ces disques sont fixés entre les deux omoplates de chaque détenu. Chacun peut observer les autres (disques) mais pas le sien. Dans la pièce, le directeur s'assure qu'il n'y a aucun miroir, et les postulants n'ont pas le droit de se communiquer la couleur des disques qu'ils voient. Le premier capable d'expliquer de quelle couleur est le disque qu'il porte sortira. On fixe aux trois concurrents un disque blanc. Les prisonniers se regardent, font quelques pas et sortent tous en même temps (idéalement) en déclarant : " Je suis blanc et voici pourquoi : j'ai vu mes deux camarades blancs ; si j'avais été noir, chacun d'eux aurait pu faire le raisonnement suivant : si j'étais noir moi aussi, le troisième sachant qu'il n'y a que deux disques noirs aurait fondu vers la sortie ; donc je ne suis pas noir. $S^{\prime}$ ils ne sont pas sortis immédiatement, c'est que je suis comme eux, blanc. » A, B et C sont les trois détenus. Si B attend la décision de $C$ et que celle-ci ne vient pas, et si B ne sort pas plus que $C$, A peut donc en déduire qu'il est blanc. Cela n'est pas exact, nous dit Lacan ; c'est bien parce que aucun des deux n'est parti en premier que cela donne à chacun à se penser blanc (première scansion). Deuxième étape. A, convaincu d'être blanc, s'en va mais les autres suivent. Si A doute d'être vu par B et $C$ noirs, il lui suffit de s'arrêter et les deux autres s'arrêtent. À ce moment-là, A peut penser que, s'il était noir, les deux autres auraient dû partir. Mais $B$ et $C$, de le voir blanc, ne le font pas ; A reprend l'initiative et tous sortent en disant qu'ils sont blancs. De cet arrêt, A ne peut tirer qu'une conclusion sans équivoque ; et c'est en cela qu'il y a progression logique. Deux scansions suspensives permettent de transformer un « sophisme menteur » en une certitude fondée en logique. Trois mouvements logiques : l'instant de voir ; le temps pour comprendre : si j'étais noir, les deux blancs que je vois ne tarderaient pas à se reconnaître comme blancs ; le moment de conclure : je me hâte de m'affirmer blanc pour que les deux autres (blancs) ne me devancent pas. Le pas logique est constitué par le troisième temps. C'est le sujet lui-même qui devient l'auteur du troisième temps dans son assertion subjective à la différence des sujets impersonnels et indéfinis réciproques des deux premiers temps : détermination temporelle de la constitution du sujet.

9. Sauf à inscrire un "passim " après le nom de Freud, nous ne pouvons que référer principalement à S. Freud, Études sur l'hystérie, 1895, Paris, PUF, 15e édition, 2002 ; Totem et tabou (1912-1913), trad. M. Weber, Paris, Gallimard, 1993 ; Malaise dans la culture, 1929, Paris, PUF, $4^{\mathrm{e}}$ édition, 2000. 
et au sentiment de culpabilité. La question reste de savoir si la réponse est celle d'un originaire, partagé par l'enfant et le primitif, ce qui fonde l'approche kleinienne de la précocité de l'œdipe et du surmoi, ou bien si cet " originaire " n'est rien d'autre que la désignation imaginaire d'un fondement symbolique, dont le poids réel est langagier.

Troisièmement, dans ses derniers travaux, et, en particulier dans Malaise dans la culture, Freud, suivant la seconde topique et l'idée du caractère primaire de la pulsion d'agression, considère alors l'image du père tyrannique de la horde primitive comme une production après-coup qui légitime la violence, devenue primaire, des frères. En quelque sorte, il réactive la dimension de l'après-coup, subvertissant une conception, linéaire cette fois, de la phylogenèse.

Lacan aborde autrement la question du temps, révélant l'implicite de la position freudienne. Dans « Le temps logique et l'assertion de certitude anticipée $^{10}$ ", l'anticipation qui mène chaque prisonnier à supposer le raisonnement des autres, pour en déduire une certitude, passe par une complexification temporelle. L'intervalle entre l'instant de voir, qui définit un présent, et le moment de conclure, actuel réalisé nécessairement plus tard, comprend un temps de latence : le temps pour comprendre. Ce dernier s'inscrit à la fois comme futur du présent et passé de cet acte «futur». Il ne fait ainsi que grammatiser la temporalité impliquée par Freud dès ses premiers travaux, le traumatisme supposant, entre sa première occurrence et son activation un temps de latence qui divise le passé comme le futur.

D'une certaine façon, la question de la temporalité, pour Lacan, y compris cette évocation du futur antérieur, ne seront, jusqu'à ses derniers enseignements, que l'explicitation de ce découpage logique du temps, entre l'instant de voir, le temps pour comprendre et le moment de conclure. Cette référence au temps logique montre, en outre, comment c'est à se poser comme objet de raisonnement de l'autre que le sujet peut anticiper la solution, et ce, non sans devoir passer par deux scansions suspensives. La topologie des nœuds et la théorie du sinthome réanimeront la question : en effet, le sinthome a cette particularité de ne pouvoir être construit qu'en second, puisqu'il vient réparer une défaillance nodale, mais, de ce fait, il est alors partie intégrante de la structure, occupant une fonction primaire : ce en quoi ses conditions de constitution, comme son dessin particulier, sont préalables à sa construction. On ne peut qu'émettre des hypothèses sur ce qu'aurait apporté le séminaire annoncé sur « La topologie et le temps » devenu... " La dissolution ».

Le temps pour comprendre, celui du passage de l'image (voir) au signifiant, au signifiant proprement dit (conclure), peut s'étirer, et le moment de

10. J. Lacan, « Le temps logique ou l'assertion de certitude anticipée », Écrits, Paris, Le Seuil, 1966. 
conclure, temps du nouage sinthomatique, peut se faire attendre. Surtout si s'impose cette possibilité de donner un sens à l'avant contenue dans le futur antérieur : temps psychiquement nécessaire en ce qu'il est logiquement nécessaire d'anticiper un dessein pour parvenir à conclure : $c^{\prime}$ est pourquoi la certitude est anticipée, souligne Lacan.

C'est sur le fond de cette double question de la langue et de la temporalité, en quelque sorte cette " grammaire du fantasme », qu'il faut revenir à leur double constitution pour concevoir l'impact de l'idée que le futur antérieur serait le temps du sujet.

Les apports lacaniens nous éclairent sur une logique temporelle articulée à la temporalité du sujet, le sujet de l'inconscient, inconscient dont Freud a repéré l'intemporalité. Si « Wo es war, soll Ich werden », on ne peut concevoir cette opération psychique fondamentale qu'en posant le sujet de l'inconscient comme marqué d'un présent, déjà passé dans l'anticipation d'un futur, lui-même sous condition d'un passé qui demeure actuel.

\section{CONSTITUTION SUBJECTIVE DE LA TEMPORALITÉ}

Pour comprendre cette valeur accordée au futur antérieur ${ }^{11}$, il convient d'en revenir, même sur un mode mythique, à la constitution de la temporalité subjective en tant qu'elle périodise ensemble sujet et altérité.

No future : à partir de ce slogan, de cette formule adolescente, s'entrevoient ces impossibilités relatives à un futur qui ne contiendrait plus que la solution perverse, le suicide ou la mort, l'anticipation de la mort. Le « surmoi collectif » évoqué par Lacan ${ }^{12}$ en 1950, ce surmoi auquel est particulièrement

11. Dans la note qui suit le Séminaire sur La lettre volée, Lacan fait une référence précise au futur antérieur : il y construit une structure symbolique à partir d'une suite aléatoire de (+) et de (-) (de l'enregistrement de piles et de faces au lancer d'une pièce); il invente un alphabet à partir du regroupement par trois des différents types de succession de résultats obtenus : signes similaires (+++ ou ---), symétries (+-+ ou -+-), dissymétries (-++, --+, ++-, +--) qu'il va nommer par exemple respectivement $(a),(b)$ et $(c)$; il fait déjà apparaître que dès lors les signes ne peuvent pas se suivre n'importe comment, qu'il y a une loi d'ordre ; puis il va regrouper par trois des séries de regroupement (trois a, trois b, trois c ; ou aba, etc.) qu'il va nommer alpha, bêta, gamma : et c'est là, au niveau de ce langage artificiel, qu'il démontre qu'un résultat (ce qui vient après un alpha ou un bêta ou un gamma) peut dépendre du résultat qui aura été obtenu quelques coups avant (au niveau des successions de a ou b ou c); de sorte qu'il démontre l'existence d'une mémoire inhérente au symbolique sans aucune subjectivité, et qui implique un futur antérieur : si tu obtiens ce résultat, à tel niveau, c'est que tu auras obtenu tel autre auparavant... dans Écrits, Paris, Le Seuil, 1966.

12. «Aucune forme donc du surmoi n'est inférable de l'individu à une société donnée. Et le seul surmoi collectif que l'on puisse concevoir exigerait une désagrégation moléculaire intégrale de la société. Il est vrai que l'enthousiasme dans lequel nous avons vu toute une jeunesse se sacrifier pour des idéaux de néant, nous fait entrevoir sa réalisation possible à l'horizon de phénomènes sociaux de masse qui supposeraient alors l'échelle universelle. » J. Lacan, "Introduction théorique aux fonctions de la psychanalyse en criminologie ", dans Écrits, Paris, Le Seuil, 1966. 
confronté l'adolescent, ce surmoi, dans sa fonction de généralisation du surmoi œdipien, " ne promeut que des idéaux de néant. "

Comment faire dans la temporalité actuelle ? Si l'adolescent est le baromètre du social (Winnicott ${ }^{13}$ ) et/ou de la culture (Le Bachelier ${ }^{14}$ ), il témoigne d'une profonde mutation du rapport du sujet à la temporalité à travers une désobjectivation du temps.

Si le temps du sujet est le futur antérieur, nous dirions qu'aujourd'hui le futur antérieur n'est plus un temps efficace pour la structuration du sujet dans notre culture. Pour avancer dans cette proposition, reprenons la question à l'origine : comment la temporalité fonde-t-elle le sujet?

Reformulons nos acquis récents ${ }^{15}$ en termes grammaticaux.

La fin de la relation transitive entre la mère et l'enfant, qu'on la nomme ainsi ou bien relation symbiotique ou encore, avec Winnicott, préoccupation maternelle primaire, fait éclater l'immédiateté du présent, puisque la réponse au sujet en « désaide » passe par la nécessité du travail de pensée de la mère et son interprétation des manifestations de cette détresse.

Cliniquement, la mère entend l'enfant pleurer mais ignore pourquoi il pleure. La « trop bonne mère » sait, toujours, immédiatement, et il n’y a ainsi nul besoin que l'enfant accède à la langue pour formuler sa demande. La « trop mauvaise mère » ne sait pas et ne veut pas savoir. La " good enough mother ${ }^{16}$ ", mère ordinaire, mère ambivalente ${ }^{17}$, demande à l'enfant du temps pour comprendre. Ce qui importe n'est pas que la mère finisse par trouver une quelconque réponse mais bien qu'elle demande ce temps à l'enfant, qu'elle pense l'enfant, qu'elle pense en particulier sa séparation d'avec l'enfant dont témoigne sa compréhension automatique devenue impossible. C'est ce qui, paradoxalement, aura l'effet d'une subjectivation.

En effet, se révèle ici pour l'enfant la nécessité d'un " détour » pour accéder à l'objet, ou, pour ce qui intéresse notre propos, l'idée d'un délai, d'une temporisation nécessaire. Pour qu'un accès à l'objet soit possible, le sujet en passe par un temps fondé du côté de l'Autre. Ce moment produit un changement de place du sujet, le barre, l'ouvre ainsi à une place de sujet de la langue, en l'occurrence de la langue maternelle. Du fait de la langue, apparaît brutalement à l'enfant qu'il y a un passé, un présent et un futur.

13. D.W. Winnicott, «L'adolescence » (1962), dans De la pédiatrie à la psychanalyse, Paris, Payot, 1992.

14. Colloque du Bachelier, "L'adolescent, baromètre de la culture », Paris, La Salpêtrière, 2007.

15. J.-J. Rassial, M. Benhaïm, J. Ahmad, B. Jacobi, « Temps, structure et psychogenèse ", L'évolution psychiatrique, 2007, 72.

16. D.W. Winnicott, De la pédiatrie à la psychanalyse, Paris, Payot, 1992.

17. M. Benhaïm, L'ambivalence de la mère, Toulouse, érès, 2001. 
Quand l'enfant reçoit, de l'Autre primordial, de l'« autorité primordiale ", nous dit Freud, cette demande de renonciation à la satisfaction (immédiate) de la pulsion ${ }^{18}$, il répond par l'angoisse et le premier sentiment de culpabilité, mais accède par là même au temps de la langue, à une complexification de la temporalité, voire à la temporalité, en même temps qu'à la fonction de la représentation, comme condition de sa pensée comme de la pensée de l'Autre. Certes, il s'agit de la première occurrence du détour qu'évoque Wallon ${ }^{19}$, comme signe de temporalisation de l'intellect, et de la première occurrence d'une inhibition structurante, repérée par les néo-piagétiens ${ }^{20}$. Mais aussi, pour ce qui nous importe ici, c'est-à-dire ce que l'on pourrait désigner comme la mise en ordre grammaticale des représentations pré-langagières, se dessinent alors un avant la détresse, qui inscrit l'écart entre le sein halluciné, celui, dit Freud, de la première tétée qui devient alors fantasmatique, assorti de sa proto-représentation, et une satisfaction reportée après le travail, même bref, d'interprétation maternelle ${ }^{21}$. Philosophiquement, on pourrait considérer que la problématique de la présence, au sens de Heidegger, du "maintenant », tel que le traduit Derrida ${ }^{22}$, est celle qui excède immédiatement la certitude alors effacée du présent.

Jusqu'où pousser cette compréhension d'une temporalité déjà grammaticale, antérieure à la fonction nominale et pronominale, le sujet et l'Autre ne se séparant que de l'effet de cette temporisation exigée ? Sans doute, faudrait-il, sans recourir nécessairement à l'innéisme que Chomsky propose comme solution, considérer l'inclusion d'une grammaire dans la construction même des représentations, ce qui irait alors dans le sens de concevoir une altérité, antérieure logiquement, celle de l'Autre avec une majuscule, à la reconnaissance de l'autre comme esprit semblable, cet Autre sans lequel pas de sujet possible. Ce qui, du point de vue analytique, suppose que cette fonction, garantie par la nomination, est déjà à l'œuvre pour la mère qui vient d'abord incarner cet Autre secourable ; autrement dit que, s'adressant à l'enfant, elle actualise sa propre aliénation, non pas à quelqu'un (le père), mais à la double fonction de l'altérité et de la temporisation.

Dans ce temps, l'Autre protecteur, Autre que nous réduirons ici au " prochain secourable » freudien ${ }^{23}$, ne répond plus sur un mode transitif, automatique, à la situation de détresse ou de désaide : il ne répond plus immédiatement à ce qui se manifeste à lui sous la figure de l'attente.

18. S. Freud, Malaise dans la culture, op. cit.

19. H. Wallon, Les origines de la pensée chez l'enfant (1945), Paris, PUF, 1989.

20. O. Houdé, Rationalité, développement et inhibition, Paris, PUF, 1995.

21. P. Aulagnier, La violence de l'interprétation, Paris, PUF, 2003.

22. J. Derrida, «La différance», dans Théorie d'ensemble, Paris, Le Seuil, 1969.

23. S. Freud, "Esquisse pour une psychologie scientifique "(1897), dans La naissance de la psychanalyse, Paris, PUF, 1973. 
Mais cette grammaire est alors très simple, puisqu'elle se réduit à distinguer passé, présent (évanescent) et futur, de façon instantanée, sur des modes purement indicatifs et impératifs et une voie où passivité et activité se confondent. Une complexification résulte de la répétition de ce cycle, et suppose alors un accès, même passif, à la langue. Peut-être, ainsi, pourrait-on considérer tout autant certains moments du développement de l'enfant comme mode d'entrée dans la multiplication des modes (conditionnel et subjonctif se distinguant progressivement) et des voies ( $l^{\prime}$ « égocentrisme » de l'enfant ne correspondant par exemple qu'à la tentative de résoudre, par la voie pronominale, l'opposition du passif et de l'actif ${ }^{24}$ ).

Ce serait compter sans l'œdipe qui, s'il n'introduit pas à la temporalité, la complexifie.

C'est l'œdipe qui organise cette complexification de la temporalité grammaticale. Il est bien sûr possible d'y inscrire le subjonctif des vœux, l'impératif du surmoi, le conditionnel de la promesse, mais aussi, ce qui importe ici, la complexification des passés et des futurs.

Étudier la temporalité de l'adolescence, c'est mettre l'accent sur deux aspects de l'œdipe : l'origine et la promesse.

En effet, posons-nous la question avec Winnicott : pourquoi l'enfant accepte-t-il les interdits œdipiens en dehors du fait qu'il ne puisse physiologiquement en réaliser les vœux ? Parce que les vœux œdipiens sont fondamentalement liés à une promesse, une promesse d'accès différé à une jouissance de même teneur que celle à laquelle il lui est demandé de renoncer pour l'heure : quand tu seras grand... quand tu auras... la promesse œdipienne vient définir la dimension même du futur antérieur : ce que nous devons avoir fait pour accéder à...

\section{ADOLESCENCE ET TEMPORALITÉ}

À l'adolescence, la promesse, donc, s'avère trompeuse. Non seulement la promesse phallique (ce rapport à l'autre auquel il a fallu renoncer) ne laisse place qu'à une satisfaction génitale partielle, mais de plus, le temps s'étire, et La Jouissance est encore renvoyée à plus tard.

L'adolescent, trompé et déçu, se retrouve dans une nécessité de réinvention. La condition de ce dépassement repose sur la fonction du surmoi culturel qui consiste à substituer à cette promesse un autre idéal, dans le social, dans la culture. Si Freud évoque dans Malaise dans la culture ${ }^{25}$ une promesse identique mais désexualisée, Lacan montre, dans Psychanalyse et

24. J.-J. Rassial, « Ôte-moi d'un doute », Le trimestre psychanalytique, t. 5, vol. 4, 1990.

25. S. Freud, Malaise dans la culture, op. cit. 
criminologie 26 , que le surmoi collectif, culturel plus précisément, ne propose aucune promesse nouvelle, sinon peut-être des idéaux de néants au sein desquels à la figure du héros se substitue la figure du martyr ${ }^{27}$.

Pour comprendre ce moment où l'adolescence est relance de la question de l'avant, du passé, arrêtons-nous un instant sur un article de Karl Abraham ${ }^{28}$.

Dans un texte de 1913, Abraham évoque quelques vignettes cliniques montrant que chez certains enfants / patients « l'insistance sur le grand-père ou la grand-mère est due à un refus du père ou de la mère ».

« Un garçon cultive le fantasme typique d'être le prince d'un royaume imaginaire. Le roi de cet empire a les qualités mêmes qu'il respecte chez son père. Par la suite, il adjoint un père à ce roi, père qu'il dote du pouvoir de créer les choses par la parole, c'est-à-dire de la toute-puissance divine. L'effort est clair : le père tout-puissant aux yeux de l'enfant est dominé par un personnage supérieur qu'il doit respecter : ainsi son omnipotence de naguère est mise en cause. »

L'adolescence est le moment où le sujet rencontre les grands-parents, en tant qu'ils viendraient en position d'Autre de l'Autre : situant que, s'il y a un Autre de l'Autre, l'Autre ne tient plus. Abraham écrit avec pertinence «qu'il n'est pas superflu de noter que la langue favorise une telle conception: Grossvater, grand-father, grand-père », osons " Grand-Autre ».

$\mathrm{Si}$, dans un premier temps, "grand-père et grand-mère ne sont pas des êtres de sang et de chair », si «le grand-père est élevé au rang d'idéal paternel », le nouvel effondrement de cette incarnation imaginaire de l'Autre à l'adolescence, introduit l'idée d'une pré-histoire familiale.

Le texte d'Abraham l'illustre par la figure d'un patient obsessionnel rempli d'hostilité à l'égard de son père et qui apprend à son analyste que " dès son enfance, son grand-père... lui était apparu comme un dieu détrôné, comme Cronos. En confrontant son grand-père détrôné et son père encore jeune, régnant, il se procurait secrètement la consolation que le père non plus ne régnerait pas éternellement, mais serait un jour détrôné à l'exemple du grand-père ».

La clinique actuelle nous montre que la question de la pré-histoire se pose d'autant plus dans des situations de rupture entre la génération, non pas des parents et des enfants, mais des parents et celle de leurs propres parents : la seconde génération vivra de façon accrue ce «traumatisme »; en témoigne

26. J. Lacan, «Introduction théorique aux fonctions de la psychanalyse en criminologie », dans Écrits, op. cit.

27. J.-J. Rassial, op. cit.

28. "Quelques remarques sur le rôle des grands-parents dans la psychologie des névroses ", CEuvres complètes, t. I, 1907-1914, Paris, Payot. 
le titre de cet ouvrage de J.-J. Moscovitz, D'où viennent les parents ? ${ }^{29}$, associé à cette non-réponse : "Ils viennent de l'expérience de l'anéantissement. »

Si « d'où viennent les enfants ? " est une question infantile, " d'où viennent les parents ? » est une question juvénile, la question adolescente type, puisque ce qui compte alors repose sur ce qu'il y avait avant le passé, avant l'événementiel du passé, autrement dit le parfait, passé simple dans la langue française, ou l'imparfait, et ce qu'il y avait avant ce passé, le plusque-parfait.

Le dépassement œdipien de la théorie sexuelle infantile, par un fantasme qui emprunte aux mythes d'une culture donnée, déplace la question " d'où viennent les enfants ? » vers celle de savoir « d'où viennent les parents ? "; c'est en cela que déjà l'œdipe, loin d'être un simple phénomène psychologique, puisqu'il s'inscrit dans une phylogenèse, ouvre le champ de l'Autre au-delà des figures parentales. Le mythe, condition de cette opération, condition de possibilité d'un lien de filiation, d'un avant à la conception, décrit un plus-que-parfait, dont la dénomination grammaticale en français est éloquente.

La première complexification du temps est celle du passé qui dans la langue française s'inscrit dans la distinction de l'imparfait, du passé simple (le parfait), du passé composé et du plus-que-parfait.

Cette complexification du passé oriente le rapport du sujet à l'histoire, en tant qu'elle constitue un en-deçà à l'enfance, désigne déjà un Autre de l'Autre parental, précisant la qualité de cet Autre «préhistorique » que Freud décèle dans la logique de l'hystérique ${ }^{30}$.

En effet, si l'enfant renonce à ses vœux œdipiens, c'est, sans doute, comme le souligne Winnicott, du fait de son impuissance à les satisfaire, mais s'il accepte cette renonciation, et peut mettre son refoulement au service de son humanisation et de sa socialisation, c'est qu'elle est assortie $\mathrm{d}^{\prime}$ une promesse d'un futur éloigné après le futur proche, qui de ce fait prend fonction d'un futur antérieur à cette promesse, comme le passé récent est aussi référé à un passé plus ancien : " quand tu seras grand, quand tu auras accompli ce qui va être exigé de toi, durant la fin de ton enfance, alors, tu auras accès à une jouissance de même poids que celle à laquelle tu dois renoncer maintenant ».

Le détour par la question des grands-parents a témoigné d'un effondrement sur la scène affective et familiale. L'école, agent d'intégration social, en témoigne sur la scène culturelle : le futur antérieur est annulé car on ne peut plus soutenir la parole prometteuse « quand tu auras fini tes études, tu travailleras », on est obligé d'y ajouter un conditionnel/subjonctif, " quand

29. J.-J. Moscovitz, D'où viennent les parents ?, Paris, L'Harmattan, 2007.

30. S. Freud, Naissance de la psychanalyse, Paris, PUF, 1973. 
tu auras fini tes études, il se pourrait que tu travailles... si tu trouves un emploi ", rien n'est moins sûr dans cette formulation qui a alors pour effet de redoubler la tromperie qui déclenchera pour certains la dépression, pour d'autres la violence.

Bien sûr le discours social tente d'amoindrir la déception dans des formulations insuffisantes à soutenir la moindre promesse, telles que : « il vaut quand même mieux être titulaire de diplômes... pour avoir plus de chances de trouver un emploi ».

Toute la période, dite de latence, exige cette complexification du temps. La tromperie déjà de cette promesse s'indique dans la substitution des modes, dans la langue française. En effet, d'une part, ce qui doit être accompli ne se réduit pas à un futur antérieur, mais correspond plutôt logiquement à un conditionnel, et, surtout, la promesse n'indique pas un futur certain, mais un subjonctif du possible. On pourrait ainsi traduire, "si tu obéis à ces contraintes qui te sont désormais imposées, alors il y aura les conditions pour que tu puisses espérer cette jouissance (ou plutôt y croire !) ».

En d'autres termes, l'œdipe est introduction d'une temporisation seconde, dont on voit bien qu'elle est ce qui échoue, de trop de lucidité quant à la valeur des promesses surmoïques, chez l'enfant engagé sur une voie psychopathique, ce qui est bien examiné, avec d'autres instruments, par Bernard Gibello ${ }^{31}$. Il avait repéré cet échec de la temporisation chez des petits sujets psychopathes, cet échec de l'acquisition des temps intermédiaires, non pas un échec radical du futur et du passé, non pas cet écrasement que l'on remarquerait peut-être dans la psychose, mais l'impossibilité de penser des temps intermédiaires. Le passé n'est rien que le passé, le présent, c'est le présent, le futur n'est rien que le futur, alors que chacun de ces temps est évidemment divisé.

Serait-on là face à une sorte de « symptôme » temporel ?

\section{CONCLUSION}

De ne pouvoir « conclure », l'adolescent passe à l'acte. Dans le temps pour comprendre, s'il est un passage possible au symbolique, il repose sur la possible confrontation du sujet à un Autre qui ne serait plus le garant absolu. De plus, conclure signerait non seulement que l'adolescent supporte ce fait humain (d'un Autre à jamais barré) mais qu'il s'en sépare, qu'il n'en dépende plus sur un mode totalitaire, pour supporter sa propre aliénation à un Autre barré. La clinique adolescente des mises en acte témoigne bien que c'est à partir d'un impossible renoncement que l'après ne réussit pas à

31. B. Gibello, L'enfant à l'intelligence troublée, Paris, Le Centurion, 1984. 
fonder un avant, autrement dit que le sujet reste souvent légitimement sourd à toute tentative de « projet » d'avenir (ritournelle institutionnelle). Les actes peuvent alors se répéter et se succéder comme seule certitude d'existence, ce pour quoi les adolescents ne peuvent en donner qu'une temporalité « hors du temps».

\section{BIBLIOGRAPHIE}

ABRAHAM, K. " Quelques remarques sur le rôle des grands-parents dans la psychologie des névroses », dans CEuvres complètes, t. I, 1907-1914, Paris, Payot.

AUlaGNIER, P. 2003. La violence de l'interprétation, Paris, PUF.

BENHAÏM, M. 2001. L'ambivalence de la mère, Toulouse, érès.

DERRIDA, J. 1968. « La différance », dans Théorie d'ensemble, Paris, Le Seuil.

FREUD, S. 1895. Études sur l'hystérie, Paris, PUF, 15e édition, 2002.

FREUD, S. 1897. «Esquisse pour une psychologie scientifique», dans La naissance de la psychanalyse, Paris, PUF, 1973.

FREUD, S. 1908. " La morale sexuelle "civilisée" et la maladie nerveuse des temps modernes ", dans La vie sexuelle, Paris, PUF, 1969.

FreUd, S. 1912-1913. Totem et tabou, trad. M. Weber, Paris, Gallimard, 1993.

FREUD, S. 1929. Malaise dans la culture, Paris, PUF, 4 e édition, 2000.

FREUD, S. 1939. L'homme Moïse et la religion monothéiste, Paris, Gallimard, 1986.

FREUD, S. 1973. Naissance de la psychanalyse, Paris, PUF.

GIBELLO, B. 1984. L'enfant à l'intelligence troublée, Paris, Le Centurion.

HeideGger, M. 1986. Être et temps, tr. fr. F. Vezin, Paris, Gallimard (7e éd. 1998).

HOUDE, O. 1995. Rationalité, développement et inhibition, Paris, PUF.

LACAN, J. 1945. « Le temps logique ou l'assertion de certitude anticipée », dans Écrits, Paris, Le Seuil, 1966.

LACAN, J. 1948. «Introduction théorique aux fonctions de la psychanalyse en criminologie », dans Écrits, Paris, Le Seuil, 1966.

LACAN, J. 1970. « Radiophonie », Scilicet 2/3, Seuil, Paris, Le Seuil.

MoscovitZ, J.-J. 2007. D'où viennent les parents ?, Paris, L'Harmattan.

RASSIAL, J.-J. 1990. « Ôte-moi d'un doute», Le trimestre psychanalytique, t. 5, vol. 4.

RASSIAL, J.-J. 1990. "Remarques sur le verlan des beurs », La psychanalyse de l'enfant, $\mathrm{n}^{\circ} 6$.

RASSIAL, J.-J. 2000. "L'espace adolescent : du monde clos à l'univers infini », dans Sortir : l'opération adolescente, Toulouse, érès.

RASSIAL, J.J. ; BENHAÏM, M. ; AHMAD, J. ; JACOBI, B. 2007. « Temps, structure et psychogenèse », L'évolution psychiatrique, 72.

WALLON, H. 1945. Les origines de la pensée chez l'enfant, Paris, PUF, 1989.

WinniCOTT, D.W. 1948. De la pédiatrie à la psychanalyse, Paris, Payot, 1992.

WinNICOTT, D.W. 1962. «L'adolescence », De la pédiatrie à la psychanalyse, Paris, Payot, 1992. 


\section{Résumé}

Si le sujet, comme le propose Lacan, est bien effet de la langue, alors l'état de la grammaire associée à la langue maternelle est en relation avec l'effet de la culture sur la subjectivation. La concordance des temps et des modes, dans une langue et à un moment donné, comme la multiplicité des modes de formulation syntaxique du passé, du présent et du futur, sont adéquats à la temporalité dans laquelle le sujet s'inscrit. Ici, l'accent sera mis sur le futur, et plus précisément sur le futur antérieur, " passé conditionnel du futur », présent en allemand comme en français, et que Heidegger, suivi par Lacan, définit comme le temps où se manifeste le sujet, comme il se manifeste aussi dans le « ne » explétif.

Mots-clés

Adolescence, grammaire, temporalité, sujet, psychanalyse.

\section{NO FUTURE}

GRAMMAR OF THE POSTMODERN SUBJECT

Summary

If the subject, as proposed by Lacan, is the effect of language, then the state of grammar associated with the maternal language is related to the effect of culture on the subjectivation. Times and modes'sequence, in a language and at one point, as the multiple modes of syntactic formulation of past, present and future, are adequate to temporality in which the topic is. Here, emphasis will be placed on the future, and more specifically on the future earlier, " conditional past of the future ", now in German as in french, and that Heidegger, followed by Lacan, defined as the time when the subject is manifested, As it manifests itself also in the « no » explétif.

Keywords

Adolescence, grammar, temporality, subject, psychoanalysis. 